SNUST 01-0502

hep-th/0107106

\title{
Anatomy of One-Loop Effective Action in Noncommutative Scalar Field Theories 1
}

\author{
Youngjai Kiem ${ }^{a}$, Soo-Jong Rey ${ }^{b, c}$, Haru-Tada Sato ${ }^{a}$, Jung-Tay Yee ${ }^{b}$ \\ BK21 Physics Research Division 83 Institute of Basic Science \\ Sungkyunkwan University, Suwon 440-746 KOREA ${ }^{a}$ \\ School of Physics 83 Center for Theoretical Physics \\ Seoul National University, Seoul 151-747 KOREA ${ }^{b}$ \\ Theory Division, CERN, CH-1211 Genevé SWITZERLAND ${ }^{c}$ \\ ykiem, haru@newton.skku.ac.kr sjrey@gravity.snu.ac.kr jungtay@phya.snu.ac.kr

\begin{abstract}
One-loop effective action of noncommutative scalar field theory with cubic self-interaction is studied. Utilizing worldline formulation, both planar and nonplanar parts of the effective action are computed explicitly. We find complete agreement of the result with Seiberg-Witten limit of string worldsheet computation and standard Feynman diagrammatics. We prove that, at lowenergy and large noncommutativity limit, nonplanar part of the effective action is simplified enormously and is resummable into a quadratic action of scalar open Wilson line operators.
\end{abstract}

PACS: 02.10.Jf, 03.65.Fd, 03.70.+k

Keywords: open wilson line, generalized star product, noncommutative scalar field theory

\footnotetext{
${ }^{1}$ Work supported in part for SJR and JTY by BK-21 Initiative in Physics (SNU-Project 2), KOSEF Interdisciplinary Research Grant 98-07-02-07-01-5, and KOSEF Leading Scientist Program, for HTS by KOSEF Brain-Pool Program, and for YK by Korea Research Foundation.
} 


\section{Introduction}

Noncommutative field theories are field theories defined on noncommutative spacetime, whose coordinates are promoted to operators:

$$
\left[x^{a}, x^{b}\right]=i \theta^{a b}
$$

and fields are multiplied in terms of the $\star$-product,

$$
A(x) \star B(y):=\exp _{\star}\left(\frac{i}{2} \partial_{x} \wedge \partial_{y}\right) A(x) B(y),
$$

implying nonlocal interactions. As such, physical aspects of these theories are suspected to be significantly different from the conventional (commutative) field theory. One of the most significant features is the phenomenon of ultraviolet-infrared (UV-IR) mixing. Motivated partly by the phenomenon, in [1], we have studied the effective action of noncommutative scalar field theories and have found that, remarkably, nonplanar part of the effective action is expressible in terms of scalar open Wilson line operators - scalar counterpart of the open Wilson lines [2, 3, 4, 5] in noncommutative gauge theories. Specifically, for $\lambda\left[\Phi^{3}\right]_{\star}$-theory, nonplanar part of the one-loop effective action is given by

$$
\Gamma_{\mathrm{np}}[\Phi]=\frac{\hbar}{2} \int \frac{\mathrm{d}^{d} k}{(2 \pi)^{d}} \Phi_{k}[\Phi] \widetilde{\mathcal{K}_{\frac{d}{2}}}(k \circ k) W_{-k}[\Phi],
$$

at low-energy, large noncommutativity limit, where

$$
\begin{aligned}
W_{k}[\Phi] & :=\mathcal{P}_{t} \int \mathrm{d}^{d} x \exp _{\star}\left(-g \int_{0}^{1} \mathrm{~d} t|\dot{y}(t)| \Phi(x+y(t))\right) \star e^{i k \cdot x} \\
\left(\Phi^{n} W\right)_{k}[\Phi] & :=\left(-\frac{\partial}{\partial g}\right)^{n} W_{k}[\Phi], \quad n=1,2,3, \cdots \quad\left(g:=\frac{\lambda}{4 m}\right)
\end{aligned}
$$

denote the scalar open Wilson line operators and $\widetilde{\mathcal{K}}$ represents 'propagator' of the state created by the open Wilson lines. See Fig. 1. In [1], much as their counterparts in noncommutative gauge theories [2, 3, 4, 5], we have also shown that the scalar open Wilson line operators are appropriate interpolating operators for 'dipoles' — weakly interacting, nonlocal objects describing excitations in noncommutative field theories. Recall that these dipoles obey socalled 'noncommutative dipole relation':

$$
\Delta x^{a}=\theta^{a b} k_{b}
$$

where $k_{a}$ and $\Delta x^{a}$ denote center-of-mass momentum and dipole moment, respectively. As such, the dipoles are ubiquitous to any noncommutative field theory, an aspect which would explain why the open Wilson line operators play prominent roles, not only in gauge theories but also in scalar field theories, in which neither gauge invariance nor gauge field is present. 


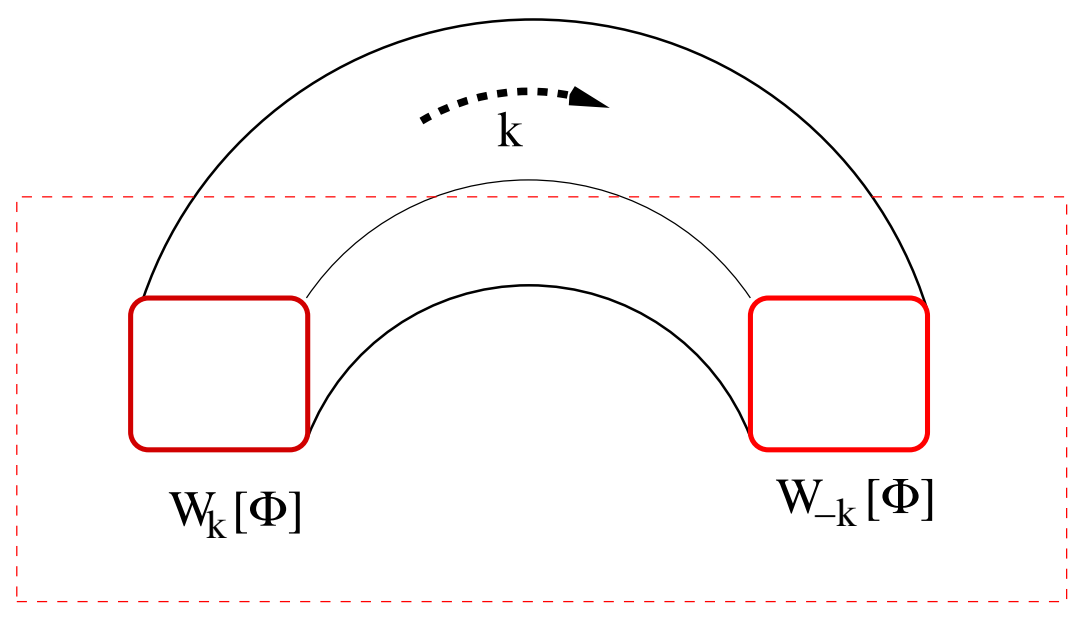

Figure 1: Scalar open Wilson line representation of nonplanar part of the one-loop effective action. The open Wilson line is an interpolating field of dipole, whose propagation is governed by the kernel $\mathcal{K}(k \circ k)$ in Eq.(1).

In this paper, in view of potential importance and far-reaching consequences of the results, Eqs.(1.1, 1.2), we present detailed computation of the one-loop effective action in $\lambda\left[\Phi^{3}\right]_{\star}$-theory. In [1], the effective action was calculated via the standard Feynman diagrammatics. To supplement the method, in this paper, we will be computing the effective action in the worldline method extended to noncommutative field theories, and make detailed comparison, wherever possible, with methods and results in the standard Feynman diagrammatics as well as in the point-particle limit of the open string worldsheet computation. For comparison, we compute both the planar and the nonplanar parts of the one-loop effective action, but in low-energy, large noncommutativity limit. In this limit, as is well-known, the Weyl-Moyal correspondence enables us to map the noncommutative $\lambda\left[\Phi^{3}\right]_{\star}$-theory in $d$ dimensions to large- $N, \mathrm{U}(N)$ matrixvalued $\lambda \operatorname{Tr}\left[\Phi^{3}\right]$-theory in $(d-2)$ dimensions. The large noncommutativity limit also allows us to recast the nonplanar part of the effective action into the form, Eq.(1.1). Somewhat surprisingly, the planar part of the one-loop effective action is not recastable in terms of closed Wilson loop operators - the putative 'master' fields in matrix-valued field theories at $N \rightarrow \infty$ limit.

This paper is organized as follows. In section 2, adopting the worldline formulation, we compute the one-loop N-point, one-particle-irreducible Green functions of noncommutative $\lambda\left[\Phi^{3}\right]_{\star}$-theory. In doing so, we also observe that noncommutative vertex operators are modified into a form showing the dipole relation Eq.(1.3) maninfestly. In section 3, we compare the result of section 2 with open string computation of N-point S-matrix amplitude at one-loop, and find a complete agreement in the Seiberg-Witten scaling limit [6]. In section 4, based on the results in sections 2 and 3, we compute the one-loop effective action, for both planar and nonplanar contributions, by summing over the N-point Green functions. Several remarks and discussions are relegated to the last section. 
Our notations are as follows. The spacetime is taken $d$-dimensional, Wick rotated to Euclidean signature, with metric $G_{a b}$. Spacetime indices are denoted $a, b, c, \cdots=1,2, \cdots, d$. Products involving successively increasing powers of the noncommutativity parameter $\theta^{a b}$ are denoted as:

$$
p \cdot q:=p_{a} G^{a b} q_{b}, \quad p \wedge q:=p_{a} \theta^{a b} q_{b}, \quad p \circ q:=p_{a}\left(-\theta^{2}\right)^{a b} q_{b} \cdots
$$

\section{$2 \lambda\left[\Phi^{3}\right]_{\star}$-Theory: Worldline Formulation}

Begin with the worldline formulation of the noncommutative $\lambda\left[\Phi^{3}\right]_{\star}$-theory. As stated in the Introduction, we are motivated to do so for detailed comparison with parallel computatons in the open string theory in the Seiberg-Witten limit. Moreover, the worldline formulation applied to noncommutative field theories, by itself, is of some interest .

\subsection{The effective action at one-loop}

The classical action of the theory is given, after Wick-rotation to Euclidean space, by

$$
S_{\mathrm{NC}}=\int \mathrm{d}^{d} x\left(\frac{1}{2}(\partial \Phi)^{2}+\frac{1}{2} m^{2} \Phi^{2}+\frac{\lambda}{3 !} \Phi \star \Phi \star \Phi\right)
$$

or, after Fourier transform, by

$$
S_{\mathrm{NC}}=\int \frac{\mathrm{d}^{d} k}{(2 \pi)^{d}} \frac{1}{2} \widetilde{\Phi}(-k)\left(k^{2}+m^{2}\right) \widetilde{\Phi}(k)+\frac{\lambda}{3 !} \int \prod_{a=1}^{3} \frac{\mathrm{d}^{d} k_{a}}{(2 \pi)^{d}} \widetilde{\Phi}\left(k_{a}\right) e^{-\frac{i}{2} \sum_{i<j} k_{i} \wedge k_{j}}(2 \pi)^{d} \delta\left(\sum_{i=1}^{3} k_{i}\right) .
$$

The effective action is evaluated most conveniently by utilizing the background field method: split the scalar field $\widetilde{\Phi}$ as $\widetilde{\Phi}=\widetilde{\Phi}_{0}+\widetilde{\varphi}$, where $\widetilde{\Phi}_{0}$ and $\widetilde{\varphi}$ denote classical (background) and quantum (internal) fields, respectively. To one-loop order, only the terms quadratic in $\tilde{\varphi}$ are relevant. Explicitly, we have

$$
\begin{aligned}
S_{\mathrm{NC}} & =\int \frac{\mathrm{d}^{d} k_{1}}{(2 \pi)^{d}} \frac{\mathrm{d}^{d} k_{2}}{(2 \pi)^{d}}\left[(2 \pi)^{d} \delta^{d}\left(k_{1}+k_{2}\right) \frac{1}{2}\left(k_{1}^{2}+m^{2}\right)\right. \\
& \left.+\frac{\lambda}{2} \int \frac{\mathrm{d}^{d} k_{3}}{(2 \pi)^{d}}(2 \pi)^{d} \delta^{d}\left(k_{1}+k_{2}+k_{3}\right) e^{-\frac{i}{2} \sum_{i<j}^{3} k_{i} \wedge k_{j}} \widetilde{\Phi}_{0}\left(k_{3}\right)\right] \widetilde{\varphi}\left(k_{1}\right) \widetilde{\varphi}\left(k_{2}\right)+\cdots
\end{aligned}
$$

It shows that, compared to commutative $\lambda\left[\Phi^{3}\right]$-theory, interaction vertices are modified by Moyal's phase-factor. These phase-factors let the scalar fields to be noncommutative while retaining associativity. We can view the scalar fields effectively as matrix-valued fields, whose precise nature is dictated by the so-called Weyl-Moyal correspondence map. Accordingly, the correspondence allows us to classify Feynman diagrams in $\lambda\left[\Phi^{3}\right]_{\star}$-theory into the planar and

\footnotetext{
${ }^{2}$ Computations below follow closely the worldline formulation of commutative field theories [7].
} 
the nonplanar ones [8, 9, 10. After symmetrization over the momentum labelling, Eq.(2.1) is re-expressible in a form suited for dealing with the planar and nonplanar diagrams:

$$
\begin{aligned}
S_{\mathrm{NC}} & =\int \frac{\mathrm{d}^{d} k_{1}}{(2 \pi)^{d}} \frac{\mathrm{d}^{d} k_{2}}{(2 \pi)^{d}}(2 \pi)^{d}\left[\frac{1}{2}\left(k_{1}^{2}+m^{2}\right) \delta^{d}\left(k_{1}+k_{2}\right)\right. \\
& \left.+\frac{\lambda}{4} \int \frac{\mathrm{d}^{d} p}{(2 \pi)^{d}} \delta^{d}\left(k_{1}+k_{2}+p\right)\left(e^{\frac{i}{2} p \wedge k_{1}}+e^{-\frac{i}{2} p \wedge k_{1}}\right) \widetilde{\Phi}_{0}(p)\right] \widetilde{\varphi}\left(k_{1}\right) \widetilde{\varphi}\left(k_{2}\right)+\cdots
\end{aligned}
$$

Integrating out the quantum fluctuation field $\widetilde{\varphi}$, the one-loop effective action is given schematically as

$$
\Gamma_{1-\text { loop }}\left[\Phi_{0}\right]=\hbar \ln \operatorname{Det}^{-\frac{1}{2}}\left[\left(k^{2}+m^{2}\right)+\frac{\lambda}{2} \int \frac{\mathrm{d}^{d} p}{(2 \pi)^{d}}\left(e^{\frac{i}{2} p \wedge k}+e^{-\frac{i}{2} p \wedge k}\right) \widetilde{\Phi}_{0}(p)\right] .
$$

Compared to the one-loop effective action of the commutative $\lambda\left[\Phi^{3}\right]$-theory:

$$
\Gamma_{1-\mathrm{loop}}\left[\Phi_{0}\right]=\hbar \ln \operatorname{Det}^{-\frac{1}{2}}\left[\left(k^{2}+m^{2}\right)+\lambda \int \frac{\mathrm{d}^{d} p}{(2 \pi)^{d}} \widetilde{\Phi}_{0}(p)\right]
$$

the interaction vertex is modified by noncommutativity in two ways. First, the coupling parameter is reduced effectively by a factor of 2 . Its combinatoric origin is as follows: the entire 3 ! diagrams can be grouped into two sets of 3 diagrams, related one another by cyclic permutations. Due to Moyal's phase-factors, they constitute inequivalent diagrams. We will refer the two respective types of interaction vertices in Eq.(2.3) as $\mathbf{P}$ and $\mathbf{T}$, respectively. Second, relative sign between $\mathbf{P}$ and $\mathbf{T}$ terms is positive. Recall that, for the vector particles as in noncommutative gauge theories, the sign is negative. In fact, these signs are attributed to even/odd parity under the worldline time-reversal $\tau \rightarrow(1-\tau)$.

For the worldline formulation, we begin with re-expressing the one-loop effective action Eq. 2.3) in path integral representation. In doing so, because of noncommutativity in $\mathbf{P}$ and $\mathbf{T}$, we will need to take care of operator-ordering. We thus start with

$$
-\ln \operatorname{Det} \mathcal{F}(k)=\int_{0}^{\infty} \frac{\mathrm{dT}}{\mathrm{T}_{x(\mathrm{~T})=x(0)}} \int_{k(\mathrm{~T})=k(0)} \mathcal{D} x(\tau) \int_{0} \mathcal{D} k(\tau) \mathcal{P}_{\tau} \exp \left(-\int_{0}^{\mathrm{T}}[\mathcal{F}(k(\tau))-i k(\tau) \cdot \dot{x}(\tau)] \mathrm{d} \tau\right) .
$$

In theories with nonderivative interactions, such as commutative $\lambda\left[\Phi^{3}\right]$-theory, the function $\mathcal{F}(k)$ is typically quadratic in $k$, and hence integration over $k(\tau)$ first would be straightforward. In the present case, due to the $k$-dependent Moyal's phase-factors in $\mathbf{P}$ and $\mathbf{T}$, we proceed differently and expand the background $\Phi_{0}$ first. The one-loop effective action then comprises of terms involving various powers of $\mathbf{P}$ 's and $\mathbf{T}$ 's, in which $\mathbf{P} \rightarrow \mathbf{T}$ is made by an insertion of 'twist' to adjacent internal lines. Explicitly,

$$
\Gamma_{1-\text { loop }}\left[\Phi_{0}\right]=\frac{\hbar}{2} \int_{0}^{\infty} \frac{\mathrm{dT}}{\mathrm{T}} \iint \mathcal{D} x \mathcal{D} k \exp \left(-\int_{0}^{\mathrm{T}} \mathrm{d} \tau\left(k^{2}+m^{2}-i k \cdot \dot{x}\right)\right)
$$




$$
\begin{aligned}
\times \sum_{\mathrm{N}=0}^{\infty} \sum_{n=0}^{\mathrm{N}}\left(-\frac{\lambda}{2}\right)^{\mathrm{N}} & {\left[\prod_{\ell=1}^{n} \int_{0}^{\tau_{\ell+1}} \mathrm{~d} \tau_{\ell} \int \frac{\mathrm{d}^{d} p_{\ell}}{(2 \pi)^{d}} \widetilde{\Phi_{0}}\left(p_{\ell}\right)\right] \exp \left(+\frac{i}{2} \sum_{\ell=1}^{n} p_{\ell} \wedge k\left(\tau_{\ell}\right)\right) } \\
\times & {\left[\prod_{j=1}^{\mathrm{N}-n} \int_{0}^{\tau_{j+1}^{\prime}} \mathrm{d} \tau_{j}^{\prime} \int \frac{\mathrm{d}^{d} p_{j}^{\prime}}{(2 \pi)^{d}} \widetilde{\Phi_{0}}\left(p_{j}^{\prime}\right)\right] \exp \left(-\frac{i}{2} \sum_{j=1}^{\mathrm{N}-n} p_{j}^{\prime} \wedge k\left(\tau_{j}^{\prime}\right)\right) . }
\end{aligned}
$$

Our notation is as follows. Each summand in Eq.(2.4) comprises of $n$ and $(\mathrm{N}-n)$ interaction vertices with(out) twists, respectively. See Fig. 2. For each group of vertices, moduli parameters are labeled as $\tau_{\ell}$ and $\tau_{j}^{\prime}\left(\tau_{n+1}=\tau_{\mathrm{N}-n+1}^{\prime}=\mathrm{T}\right)$, and external momenta are labeled as $p_{\ell}$ and $p_{j}^{\prime}$, respectively. We also assign sign factor $\nu_{l}=+1,-1$ to these two groups of interaction vertices. The two square brackets in Eq. 2.4) are untwisted and twisted interaction vertices, respectively. Therefore, for given $n$ and $\mathrm{N}$, the one-loop diagram is a function of the following set of momenta and moduli parameters:

$$
\begin{aligned}
& \left\{\tau_{i}\right\}=\left\{\tau_{(l)} \text { for } \quad i=1,2, \cdots, n ; \quad \tau_{(j)}^{\prime} \quad \text { for } \quad i=n+1, \cdots, \mathrm{N}\right\} \\
& \left\{p_{i}\right\}=\left\{p_{(l)} \text { for } i=1,2, \cdots, n ; \quad p_{(j)}^{\prime} \quad \text { for } \quad i=n+1, \cdots, \mathrm{N}\right\} .
\end{aligned}
$$

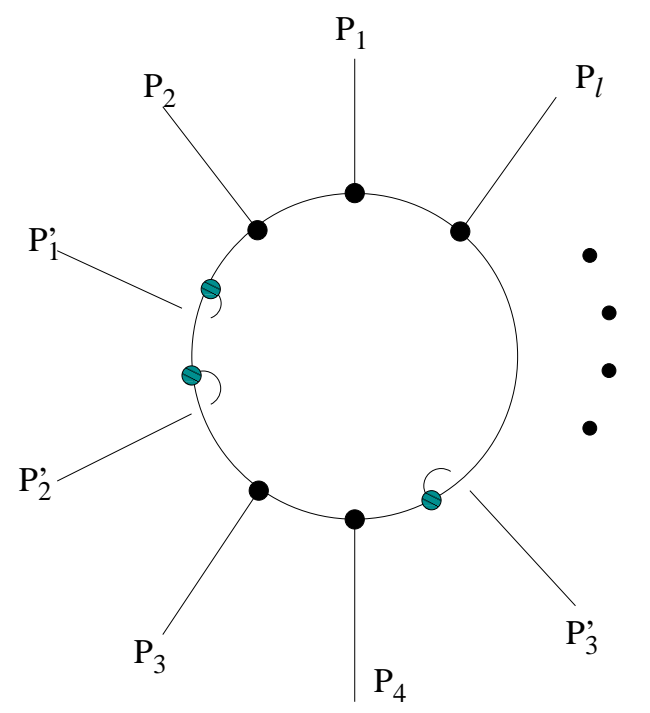

Figure 2: One-Loop N-point Green function. Interaction vertices of untwisted and twisted types are marked with solid and dashed circles, whose momenta are labelled as $p_{1}, \cdots, p_{\ell}$ and $p_{1}^{\prime}, \cdots, p_{\mathrm{N}-n}^{\prime}$.

The N-point, one-particle-irreducible (1PI), Green functions are obtained by expanding the effective action Eq.(2.4) in powers of $\Phi_{0}$. In the commutative setup, they are calculated by substituting the classical background field into a sum of 'plane-wave':

$$
\widetilde{\Phi_{0}}(x(\tau)) \longrightarrow \sum_{\ell=1}^{\mathrm{N}} e^{i p_{\ell} \cdot x(\tau)} .
$$


This substitution is still valid for the present case, as the products between $\widetilde{\Phi_{0}}$ 's in (2.4) in the momentum represetation are local products (with explicit Moyal's phase-factors attached). By making the plane-wave substitution Eq.(2.5), we now generate all the possible diagrams automatically (discarding terms containing the same momentum). This leads, for given $n$ and $\mathrm{N}$, the moduli integrals in Eq.(2.4) to

$$
\begin{gathered}
{\left[\prod_{\ell=1}^{n} \int_{0}^{\tau_{\ell+1}} \mathrm{~d} \tau_{\ell} \widetilde{\Phi_{0}}\left(p_{\ell}\right)\right] \cdot\left[\prod_{j=1}^{\mathrm{N}-n} \int_{0}^{\tau_{j+1}^{\prime}} \mathrm{d} \tau_{j}^{\prime} \widetilde{\Phi_{0}}\left(p_{j}^{\prime}\right)\right]} \\
\longrightarrow \prod_{\ell=1}^{n} \int_{0}^{\tau_{\ell+1}} \mathrm{~d} \tau_{\ell} \prod_{j=1}^{\mathrm{N}-n} \int_{0}^{\tau_{j+1}^{\prime}} \mathrm{d} \tau_{j}^{\prime}\left[e^{i p_{1} x\left(\tau_{1}\right)} e^{i p_{2} x\left(\tau_{2}\right)} \cdots e^{i p_{\mathrm{N}} x\left(\tau_{\mathrm{N}}\right)}\right. \\
+(\text { all permutations })]
\end{gathered}
$$

By interchanging moduli variables $\tau$ 's, all permutation terms in Eq.(2.6) can be arranged as all possible ordered integrals having the same integrand. We find that the right-hand side of Eq.(2.6) involves the moduli-space integrals:

$$
\sum_{\left\{\nu_{i}\right\}} \int_{0}^{\mathrm{T}} \mathrm{d} \tau_{\mathrm{N}} \cdots \int_{0}^{\mathrm{T}} \mathrm{d} \tau_{1} \prod_{\ell=1}^{\mathrm{N}} e^{i p_{\ell} x\left(\tau_{\ell}\right)}=\sum_{\left\{\nu_{i}\right\}} \frac{\mathrm{T}}{\mathrm{N}} \int_{0}^{\mathrm{T}} \mathrm{d} \tau_{\mathrm{N}-1} \cdots \int_{0}^{\mathrm{T}} \mathrm{d} \tau_{1} \prod_{\ell=1}^{\mathrm{N}} e^{i p_{\ell} x\left(\tau_{\ell}\right)}
$$

This is essentially the N-point correlator (evaluated with an appropriate worldline Green function). The combinatorics work as follows. The sum over $\left\{\nu_{i}\right\}$ takes into account of all possible $2^{N}$ terms, viz. the binomial expansion of $(\mathbf{P}+\mathbf{T})^{\mathrm{N}}$ interaction vertices. In the commutative limit, all the $2^{\mathrm{N}}$ terms reduce to the same contribution, and cancel eventually the $(1 / 2)^{N}$ factor originating from the rescaling of the coupling parameter, $\lambda \rightarrow \lambda / 2$. Alternatively, as the second second expression in the above moduli-space integral shows, the sum over $\left\{\nu_{i}\right\}$ takes into account of all possible $2^{\mathrm{N}}$-terms: $2 \mathrm{~N}$ possibilities for the $\mathrm{N}$-th reference interaction vertex, and $2^{\mathrm{N}-1}$ combinatoric possibilities for the rest. The factor of $\mathrm{N}$ is cancelled by the symmetry factor for the reference vertex, $1 / \mathrm{N}$. The net result is $2^{\mathrm{N}}$, yielding the same combinatoric counting.

Consequently, at one-loop, the N-point Green function (corresponding to Eq.(2.4)) is given by:

$$
\begin{aligned}
\Gamma_{\mathrm{N}}\left[p_{1}, \cdots, p_{\mathrm{N}}\right] & =\frac{\hbar}{2}\left(-\frac{\lambda}{2}\right)^{\mathrm{N}} \sum_{\left\{\nu_{i}\right\}} \int_{0}^{\infty} \frac{\mathrm{d} \mathrm{T}}{\mathrm{T}} \int_{0}^{\mathrm{T}} \cdots \int_{0}^{\mathrm{T}} \prod_{\ell=1}^{\mathrm{N}} \mathrm{d} \tau_{\ell} \\
& \times \int \mathcal{D} x \int \mathcal{D} k e^{-\int_{0}^{\mathrm{T}} \mathrm{d} \tau\left[k^{2}+m^{2}-i k \cdot \dot{x}\right]} \prod_{j=1}^{\mathrm{N}} e^{i p_{j} \cdot x\left(\tau_{j}\right)} e^{\frac{i}{2} \nu_{j} p_{j} \wedge k\left(\tau_{j}\right)} .
\end{aligned}
$$

A brief comment is in order. In the above derivation, for simplicity, we have utilized the plane-wave basis. As will be shown momentarily, the phase-factor $\exp \left[\frac{i}{2} \nu_{j} p_{j} \wedge k\left(\tau_{j}\right)\right]$ ought to 
be understood as part of a generalized vertex operator, viz. the plane-wave (scalar) vertex operator $\int \mathrm{d} \tau_{\ell} e^{i p_{\ell} \cdot x\left(\tau_{\ell}\right)}$ is not the proper one in noncommutative field theories. In fact, we will be showing that the standard Feynman diagrammatics results in Appendix A are reproducible precisely in terms of these new vertex operators .

\subsection{The N-point Green Function}

We next evaluate the path integral in Eq.(2.7) explicitly and derive parametric expression for the one-loop, N-point Green function. In this section, we will prove that the result is given by

$$
\begin{aligned}
\Gamma_{\mathrm{N}}\left[p_{1}, \cdots, p_{\mathrm{N}}\right]= & \frac{\hbar}{2}\left(-\frac{\lambda}{2}\right)^{\mathrm{N}} \sum_{\left\{\nu_{i}\right\}} \int_{0}^{\infty} \frac{\mathrm{dT}}{\mathrm{T}} e^{-m^{2} \mathrm{~T}}\left(\frac{1}{4 \pi \mathrm{T}}\right)^{\frac{d}{2}} \int_{0}^{\mathrm{T}} \prod_{\ell=1}^{\mathrm{N}} \mathrm{d} \tau_{\ell} \prod_{i<j}^{\mathrm{N}} e^{\frac{i}{2} \nu_{i j} \varepsilon\left(\tau_{i j}\right) p_{i} \wedge p_{j}} \\
& \times \exp \left[\frac{1}{2} \sum_{k, \ell=1}^{\mathrm{N}} p_{k} \cdot \mathcal{G}_{B \theta}\left(\tau_{k}, \tau_{\ell} ; \varepsilon_{k}, \varepsilon_{\ell}\right) \cdot p_{\ell}\right]
\end{aligned}
$$

where $\mathcal{G}_{B \theta}^{a b}$ denotes noncommutative counterpart of the worldline propagator $\mathcal{G}_{B}$ :

$$
\mathcal{G}_{B \theta}^{a b}\left(\tau_{k}, \tau_{\ell} ; \varepsilon_{k}, \varepsilon_{\ell}\right)=g^{a b} \mathcal{G}_{B}\left(\tau_{k}, \tau_{\ell}\right)-\frac{i}{\mathrm{~T}} \theta^{a b} \varepsilon_{k \ell}\left(\tau_{k}+\tau_{\ell}\right)+\frac{1}{4 \mathrm{~T}}\left(-\theta^{2}\right)^{a b} \varepsilon_{k \ell}^{2} .
$$

We have introduced the following notations

$$
\nu_{i j}:=\frac{\nu_{i}+\nu_{j}}{2}, \quad \tau_{i j}:=\tau_{i}-\tau_{j}, \quad \varepsilon_{i}:=\frac{1-\nu_{i}}{2}, \quad \text { and } \quad \varepsilon(\tau):=\operatorname{sign}(\tau) .
$$

In addition, $\varepsilon_{k \ell}$ refers to $\varepsilon_{k \ell}=\varepsilon_{k}-\varepsilon_{\ell}$. In the present case, as Eq.(2.4) indicates, the untwisted and the twisted vertices ought to be distinguished from each other, as the exponent of the

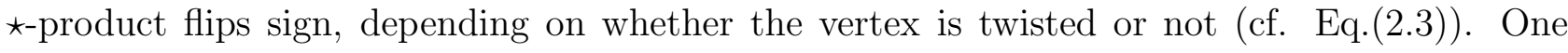
recognizes also that the $\star$-product structure is dressed with twist-dependent 'weight':

$$
e^{i p_{i} x\left(\tau_{i}\right)} *^{\nu} e^{i p_{j} x\left(\tau_{j}\right)}=\exp \left(\frac{i}{2} \nu_{i j} \varepsilon\left(\tau_{i j}\right) p_{i} \wedge p_{j}\right) e^{i p_{i} x\left(\tau_{i}\right)+i p_{j} x\left(\tau_{j}\right)},
$$

where $\nu_{i j}=0,+1,-1$ depending on types of boundaries along which the interaction vertices are inserted.

In the rest of this subsection, we prove Eqs.(2.8, 2.9). Start with the path-integral over $k(\tau)$. The relevant integral is

$$
K:=\int \mathcal{D} k \exp \left(-\int_{0}^{\mathrm{T}} \mathrm{d} \tau\left[k^{2}(\tau)-i k \cdot \dot{x}(\tau)+\frac{i}{2} \sum_{\ell=1}^{\mathrm{N}} \nu_{\ell} \delta\left(\tau-\tau_{\ell}\right) k(\tau) \wedge p_{\ell}\right]\right)
$$

a Gaussian type integral. After shifting the momentum density as

$$
k^{a}(\tau) \quad \longrightarrow \quad k^{a}(\tau)+\frac{i}{2} \dot{x}^{a}(\tau)-\frac{i}{4} \sum_{\ell=1}^{\mathrm{N}} \delta\left(\tau-\tau_{\ell}\right) \nu_{\ell} \theta^{a b} p_{\ell}^{b},
$$


the integral yields

$$
K=\mathcal{N}(\mathrm{T}) \exp \left(-\frac{1}{4} \int_{0}^{\mathrm{T}} \mathrm{d} \tau\left[\dot{x}-\frac{1}{2} \sum_{\ell=1}^{\mathrm{N}} \theta \cdot p_{\ell} \nu_{\ell} \delta\left(\tau-\tau_{\ell}\right)\right]^{2}\right),
$$

where $\mathcal{N}(\mathrm{T})$ is a $\mathrm{T}$-dependent normalization factor, which turns out the same as the commutative case. Square of the $\delta$-functions in the exponent does not lead to divergences, as all $\tau_{\ell}$ take different values because of the noncommutativity. As such, one finally finds

$$
K=\mathcal{N}(\mathrm{T}) \exp \left(-\frac{1}{4} \int_{0}^{\mathrm{T}} \dot{x}^{2} \mathrm{~d} \tau\right) \prod_{\ell=1}^{\mathrm{N}} \exp \left(\frac{1}{4} \nu_{\ell} \dot{x}\left(\tau_{\ell}\right) \wedge p_{\ell}\right) .
$$

Utilizing the expression $K$, the N-point correlation function is then reduced to

$$
\begin{aligned}
\Gamma_{\mathrm{N}}\left[p_{1}, \cdots, p_{\mathrm{N}}\right] & =\frac{1}{2}\left(-\frac{\lambda}{2}\right)^{\mathrm{N}} \sum_{\left\{\nu_{i}\right\}} \int_{0}^{\infty} \frac{\mathrm{dT}}{\mathrm{T}} e^{-m^{2} \mathrm{~T}} \int_{0}^{\mathrm{T}} \prod_{\ell=1}^{\mathrm{N}} \mathrm{d} \tau_{\ell} \\
& \times \mathcal{N}(\mathrm{T}) \int_{x(0)=x(\mathrm{~T})} \mathcal{D} x \exp \left(-\frac{1}{4} \int_{0}^{\mathrm{T}} \dot{x}^{2} \mathrm{~d} \tau\right) \prod_{\ell=1}^{\mathrm{N}} e^{i p_{\ell} x\left(\tau_{\ell}\right)} e^{\frac{1}{4} \dot{x}\left(\tau_{\ell}\right) \wedge p_{\ell} \nu_{\ell}} .
\end{aligned}
$$

Next, evaluate the path integral over $x(\tau)$ :

$$
X:=\int_{x(0)=x(\mathrm{~T})} \mathcal{D} x e^{-\frac{1}{4} \int_{0}^{\mathrm{T}} \dot{x}^{2} d \tau} \prod_{\ell=1}^{\mathrm{N}} \exp \left(i p_{\ell} \cdot x\left(\tau_{\ell}\right)-\frac{\nu_{\ell}}{4} p_{\ell} \wedge \dot{x}\left(\tau_{\ell}\right)\right) .
$$

The integrand suggests that the vertex operator relevant for the noncommutative scalar field is not the conventional plane-wave vertex operator but, as mentioned earlier,

$$
V_{\mathrm{NC}}(x):=\int_{0}^{\mathrm{T}} \mathrm{d} \tau \exp \left(i p \cdot x(\tau)-\frac{\nu}{4} p \wedge \dot{x}(\tau)\right) .
$$

The integral $X$ is evaluated as follows. First, decompose the $x(\tau)$ field into normal modes:

$$
x^{\mu}(\tau)=x_{0}^{\mu}+\sum_{n=1}^{\infty} x_{n}^{\mu} \sin \left(\frac{n \pi \tau}{\mathrm{T}}\right) .
$$

Integral over the zero-mode $x_{0}$ enforces total energy-momentum conservation. The rest yields

$$
X=\int_{-\infty}^{\infty} \prod_{n=1}^{\infty} \mathrm{d} x_{n} \exp \left[-\frac{\pi^{2}}{8 \mathrm{~T}} n^{2} x_{n}^{2}+i \sum_{\ell=1}^{\mathrm{N}} p_{\ell} x_{n} \sin \left(\frac{n \pi \tau_{\ell}}{\mathrm{T}}\right)-\frac{1}{4} \sum_{\ell=1}^{\mathrm{N}} p_{\ell} \wedge x_{n} \nu_{\ell} \frac{n \pi}{\mathrm{T}} \cos \left(\frac{n \pi \tau_{\ell}}{\mathrm{T}}\right)\right] .
$$

The $x_{n}$ integrations are of Gaussian type. Completing the exponent into squares and fixing the normalization as in the commutative case, we obtain

$$
X=\left(\frac{1}{4 \pi \mathrm{T}}\right)^{\frac{d}{2}} \prod_{n=1}^{\infty} \exp \left[\frac{2 \mathrm{~T}}{n^{2} \pi^{2}}\left(i \sum_{\ell=1}^{\mathrm{N}} p_{\ell} \sin \left(\frac{n \pi \tau_{\ell}}{\mathrm{T}}\right)+\frac{1}{4} \sum_{\ell=1}^{\mathrm{N}} \theta \cdot p_{\ell} \nu_{\ell} \frac{n \pi}{\mathrm{T}} \cos \left(\frac{n \pi \tau_{\ell}}{\mathrm{T}}\right)\right)^{2}\right] .
$$


Applying the identities

$$
\sin \left(\frac{n \pi \tau_{i}}{\mathrm{~T}}\right) \sin \left(\frac{n \pi \tau_{j}}{\mathrm{~T}}\right)=\frac{1}{2}\left(\cos \frac{n \pi\left(\tau_{i}-\tau_{j}\right)}{\mathrm{T}}-\cos \frac{n \pi\left(\tau_{i}+\tau_{j}\right)}{\mathrm{T}}\right), \quad \text { etc. }
$$

and

$$
\begin{aligned}
& \sum_{n=1}^{\infty} \frac{\cos n x}{n^{2}}=\frac{1}{4}(|x|-\pi)^{2}-\frac{\pi^{2}}{12} \\
& \sum_{n=1}^{\infty} \cos n(x-a)=\pi \delta(x-a)-\frac{1}{2},
\end{aligned}
$$

we obtain

$$
\begin{aligned}
X=\left(\frac{1}{4 \pi \mathrm{T}}\right)^{\frac{d}{2}} \exp [ & -\frac{\mathrm{T}}{4} \sum_{i, j=1}^{\mathrm{N}} p_{i} \cdot p_{j}\left\{\left(1-\frac{\left|\tau_{i}-\tau_{j}\right|}{\mathrm{T}}\right)^{2}-\left(1-\frac{\tau_{i}+\tau_{j}}{\mathrm{~T}}\right)^{2}\right\} \\
& +\frac{i}{8} \sum_{i, j=1}^{\mathrm{N}} p_{i} \wedge p_{j} \nu_{j} \mathrm{~T} \frac{\partial}{\partial \tau_{j}}\left\{\left(1-\frac{\left|\tau_{i}-\tau_{j}\right|}{\mathrm{T}}\right)^{2}-\left(1-\frac{\tau_{i}+\tau_{j}}{\mathrm{~T}}\right)^{2}\right\} \\
& \left.-\frac{1}{4 \mathrm{~T}} \sum_{i, j=1}^{\mathrm{N}} p_{i} \circ p_{j} \varepsilon_{i} \varepsilon_{j}\right]
\end{aligned}
$$

where $\delta\left(\tau_{i} \pm \tau_{j}\right)=0$ is used again. The differentiation with respect to $\tau_{j}$ in the second line of Eq.(2.11) produces both the Filk phase-factor and the terms linear in $\tau$ 's, which will be shown to yield precisely the generalized $\star$-products. Making use of the identities derived from the energy-momentum conservation:

$$
\begin{aligned}
\sum_{i, j=1}^{\mathrm{N}} p_{i} \wedge p_{j} \nu_{j} \tau_{i} & =\sum_{i, j=1}^{\mathrm{N}} p_{i} \wedge p_{j} \varepsilon_{i j}\left(\tau_{i}+\tau_{j}\right), \\
\sum_{i, j=1}^{\mathrm{N}} p_{i} \circ p_{j} \varepsilon_{i} \varepsilon_{j} & =-\frac{1}{2} \sum_{i, j=1}^{\mathrm{N}} \varepsilon_{i j}^{2} p_{i} \circ p_{j},
\end{aligned}
$$

we were able to arrange the $X$-integral as

$$
\begin{aligned}
X & =\left(\frac{1}{4 \pi \mathrm{T}}\right)^{\frac{d}{2}} \exp \left[\frac{i}{2} \sum_{i<j}^{\mathrm{N}} p_{i} \wedge p_{j} \nu_{i j} \varepsilon\left(\tau_{i j}\right)\right] \\
& \times \exp \left[\frac{1}{2} \sum_{i, j=1}^{\mathrm{N}} p_{i} \cdot p_{j} \mathcal{G}_{B}\left(\tau_{i}, \tau_{j}\right)-\frac{i}{2 \mathrm{~T}} \sum_{i, j=1}^{\mathrm{N}} p_{i} \wedge p_{j} \varepsilon_{i j}\left(\tau_{i}+\tau_{j}\right)+\frac{1}{8 \mathrm{~T}} \sum_{i, j=1}^{\mathrm{N}} \varepsilon_{i j}^{2} p_{i} \circ p_{j}\right] .
\end{aligned}
$$

Putting the above result into Eq.(2.10), we finally obtain the aforementioned expression, Eq.(2.8), for the N-point Green functions at one-loop order.

The N-point Green functions, Eq.(2.8), can also be obtained from rearranging the standard Feynman diagrammatics. This is elaborated in Appendix A. In comparing the two results, one should exercise a caution that, upon reversing the orientation of the underlying string worldsheet, one also flips the overall sign of the phase-factor in Eq.(2.8). In fact, the the overall sign choice is fixed only after the orientation convention is chosen. 


\section{Comparison with String Worldsheet Computation}

Having found the N-point Green functions in the worldline formalism, we now compare Eqs.(2.8, 2.9) with those obtained from the string theory computation [13]. At one-loop, the relevant string worldsheet diagram is an annulus with two boundaries. We will parametrize the worldsheet by complex coordinate, $z=x+i y$, where $y$ is periodically identified as $y \simeq y+t$ and the two boundaries are located at $x=0(\varepsilon=1$ and $\nu=-1$, the inner boundary) and $x=1 / 2(\varepsilon=0$ and $\nu=1$, the outer boundary), respectively. Here $t$ is the annulus modulus. External open strings can be inserted along either of the two boundaries, a direct counterpart of the twisted and untwisted interaction insertions in one-loop Feynman diagrammatics.

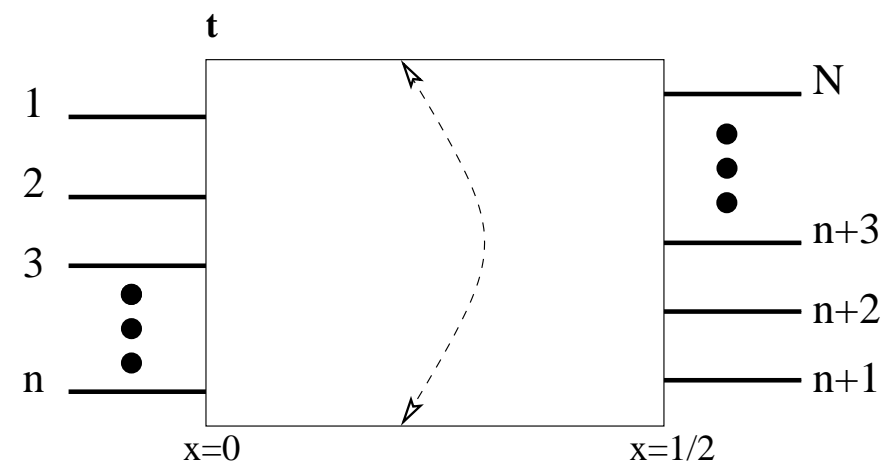

Figure 3: Annulus as one-loop string worldsheet diagram. Tachyon vertex operators are inserted on either boundary of the annulus. In the Seiberg-Witten limit, the annulus size modulus $t$ is scaled to infinity.

As we want to extract information concerning noncommutative scalar field theories, the relevant external string states are those of tachyons, whose vertex operator is given by

$$
V_{T}(p, y)=g_{s} \sqrt{\alpha^{\prime}} e^{i p \cdot X(y)}
$$

and we turn on the constant background two-form gauge fields, which turns itself into the noncommutativity parameter $\theta$ in the Seiberg-Witten limit. The relevant N-point tachyon S-matrix amplitude, which is depicted in Figure 3, is schematically expressible as (up to normalization)

$$
\begin{aligned}
\mathcal{A} & =\int_{0}^{\infty} \frac{\mathrm{d} t}{t} Z(t) \int_{0}^{t} \mathrm{~d} y_{1} \cdots \int_{0}^{t} \mathrm{~d} y_{\mathrm{N}}\left\langle V_{T 1}\left(p_{1}, y_{1}\right) \cdots V_{T \mathrm{~N}}\left(p_{\mathrm{N}}, y_{\mathrm{N}}\right)\right\rangle_{t} \\
& =\left(g_{s}^{2} \alpha^{\prime}\right)^{\frac{\mathrm{N}}{2}} \int_{0}^{\infty} \frac{\mathrm{d} t}{t} Z(t) \int_{0}^{t} \mathrm{~d} y_{1} \cdots \int_{0}^{t} \mathrm{~d} y_{\mathrm{N}} \exp \left(-\alpha^{\prime} \sum_{i<j}^{\mathrm{N}} p_{i} \mathcal{G}^{i j} p_{j}\right),
\end{aligned}
$$

in terms of the worldsheet partition function $Z(t)$ and the worldsheet Green function $\mathcal{G}^{i j}$. In the case of the annulus partition function, nonzero two-form $B_{m n}$ background does not change 
the worldsheet Green function, except that the metric is replaced, in the Seiberg-Witten limit, by the open string metric $G_{a b}$ :

$$
Z(t)=\int \frac{\mathrm{d}^{d} k}{(2 \pi)^{d}} \sum_{\{\mathrm{I}\}} e^{-2 \pi \alpha^{\prime} t\left(k \cdot G \cdot k+M_{\mathrm{I}}^{2}\right)}=\left(\frac{1}{2 \pi \alpha^{\prime} t}\right)^{\frac{d}{2}} f_{1}(q)^{-24},
$$

where the summation $\{\mathrm{I}\}$ is over the entire string states in the intermediate channel, and

$$
f_{1}(q)=q^{1 / 24} \prod_{m=1}^{\infty}\left(1-q^{m}\right) \quad \text { where } \quad q=e^{-2 \pi t},
$$

as are relevant for the bosonic $D_{(d-1)}$-branes.

The boundary worldsheet propagator has the following form [14: for two points on the same boundary, relevant for the planar diagram contributions,

$$
\mathcal{G}_{\mathrm{p}}^{a b}\left(z_{i}, z_{j}\right)=\alpha^{\prime} G^{a b} \mathcal{G}\left(z_{i}, z_{j}\right)+\frac{i}{2} \theta^{a b} \varepsilon\left(z_{i}-z_{j}\right)
$$

while for two points on different boundaries, relevant for the nonplanar diagram contributions,

$$
\mathcal{G}_{\mathrm{np}}^{a b}\left(z_{i}, z_{j}\right)=\alpha^{\prime} G^{a b} \mathcal{G}\left(z_{i}, z_{j}\right)+\frac{(\theta \cdot G \cdot \theta)^{a b}}{2 \pi \alpha^{\prime} t}\left(x_{i}-x_{j}\right)^{2}-\frac{2 i}{t} \theta^{a b}\left(x_{i}-x_{j}\right)\left(y_{i}+y_{j}\right) .
$$

Here, the function $\mathcal{G}\left(z_{i}, z_{j}\right)$ is defined by

$$
\mathcal{G}\left(z_{i}, z_{j}\right)=-\log \left|\frac{\vartheta_{1}\left(z_{i}-z_{j} \mid i t\right)}{\vartheta_{1}^{\prime}(0 \mid i t)}\right|^{2}+\frac{2 \pi}{t}\left(y_{i}-y_{j}\right)^{2},
$$

in terms of the theta function $\vartheta_{1}$.

To extract the noncommutative scalar field theory amplitudes from open string tachyon Smatrix amplitudes, we will take the Seiberg-Witten decoupling limit: $\alpha^{\prime} \rightarrow 0$ under which the massive string modes decouple, while open string metric $G^{a b}$ and noncommutativity $\theta^{a b}$ are held fixed. In fact, technically speaking, what we really do here is to isolate the loop contribution from the tachyon intermediate state. This contribution is exponentially divergent and dominates contributions from higher mass intermediate states. We then analytically continue the mass parameter $m^{2}$ to a proper positive value to match our cubic field theory mass paramenter. In this process, we also keep the field theory moduli parameters $\mathrm{T}$ and $\tau$ fixed by putting

$$
2 \pi \alpha^{\prime} t=\mathrm{T} \quad \text { and } \quad 2 \pi \alpha^{\prime} y=\tau
$$

viz. the annulus becomes infinitely thin, making essentially a circle, relevant for one-loop Feynman diagram. Through this procedure, one finds that the partition function $Z(t)$ turns into

$$
Z(t) \rightarrow\left(\frac{1}{\mathrm{~T}}\right)^{\frac{d}{2}} e^{-m^{2} \mathrm{~T}}
$$


matching the corresponding factor in the field theory result, Eq. 2.8).

The two-point function $\mathcal{G}$ in the decoupling limit is reduced to (see for instance [15]):

$$
-\alpha^{\prime} \mathcal{G}\left(z_{i}, z_{j}\right) \longrightarrow \mathcal{G}_{B}=\left|\tau_{i}-\tau_{j}\right|-\frac{\left(\tau_{i}-\tau_{j}\right)^{2}}{\mathrm{~T}}
$$

viz. only zero-mode part of $\vartheta_{1}$ remains. Also, noting that $\left(x_{i}-x_{j}\right)=-\varepsilon_{i j} / 2$ vanishes when the $i$ and $j$-th insertions are on the same boundary, the last two terms in the nonplanar propagator Eq.(3.2) are reduced to the last two terms in Eq.(2.9). Likewise, the second term in the planar propagator Eq.(3.1) gives rise to the Filk phase-factors, as, when $i$ and $j$-th insertions are along the same boundary, $\varepsilon\left(z_{i}-z_{j}\right)=\varepsilon\left(\tau_{i j}\right)$ at $x=0(\nu=-1)$ and $\varepsilon\left(z_{i}-z_{j}\right)=-\varepsilon\left(\tau_{i j}\right)$ at $x=1 / 2$ $(\nu=1)$. Putting these observations together, we conclude that Eq.(2.8) and Eq.(2.9) follows precisely from the string theory computation in the Seiberg-Witten limit.

The expression Eq. (2.8) is the general one for a given value of $\mathrm{N}$, the total number of external scalar insertions; the sum over $\left\{\nu_{i}\right\}$ is over $2^{\mathrm{N}}$ possible terms, spanning the cases of inner or outer boundary insertion for each interaction vertex. Decomposing $\mathrm{N}=\mathrm{N}_{1}+\mathrm{N}_{2}$ where $\mathrm{N}_{1}$ is the number of inner boundary insertions and $\mathrm{N}_{2}$ is the number of outer boundary insertions, the terms of Eq.(2.8) can be classified into planar and nonplanar contributions, depending on the value of $\mathrm{N}_{1}$ : two terms, $\mathrm{N}_{1}=0$ or $\mathrm{N}$, are planar diagrams, while $0<\mathrm{N}_{1}<\mathrm{N}$ are nonplanar diagrams (consisting of $\mathrm{N} ! /\left(\mathrm{N}_{1} ! \mathrm{N}_{2} !\right)$ symmetrization of the external momenta). The nonplanar diagrams correspond to the double trace terms

$$
\operatorname{Tr} \underbrace{\Phi\left(p_{1}\right) \cdots \Phi\left(p_{\mathrm{N}_{1}}\right)}_{\mathrm{N}_{1}} \operatorname{Tr} \underbrace{\Phi\left(p_{\mathrm{N}_{1}+1}\right) \cdots \Phi\left(p_{\mathrm{N}}\right)}_{\mathrm{N}_{2}} .
$$

For fixed $\mathrm{N}_{1}$, let us denote the net momentum flow between the inner and the outer boundaries as

$$
P=\sum_{i=1}^{\mathrm{N}} \varepsilon_{i} p_{i}=\sum_{r=1}^{\mathrm{N}_{1}} p_{r}
$$

From here on, the indices $r, s, \cdots$ runs from 1 to $\mathrm{N}_{1}$ (inner insertions) while the indices $m, n, \cdots$ runs from 0 to $\mathrm{N}_{2}$ (outer insertions). Using the the overall momentum conservation, we find that the contribution to the amplitudes from the third term of Eq.(2.9) can be written as

$$
\exp \left(-\frac{P \circ P}{4 \mathrm{~T}}\right)
$$

Another useful identity that can also be proved using the momentum conservation is

$$
\frac{1}{2} \sum_{i, j=1}^{\mathrm{N}} p_{i} \wedge p_{j} \varepsilon_{i j}\left(\tau_{i}+\tau_{j}\right)=\frac{1}{2} \sum_{i<j}^{\mathrm{N}} p_{i} \wedge p_{j}\left(\nu_{i}+\nu_{j}\right) \tau_{i j}
$$


The quantity $\left(\nu_{i}+\nu_{j}\right) / 2$ equals to +1 when $i$ and $j$ are both outer insertions, -1 when they are both inner insertions, and 0 otherwise. Thus, for fixed $\mathrm{N}_{1}$, each term in Eq. 2.8) can be expressed as

$$
\begin{aligned}
\Gamma_{\mathrm{N},\left\{\nu_{j}\right\}}= & \frac{\hbar}{2}\left(-\frac{\lambda}{2}\right)^{\mathrm{N}} \int_{0}^{\infty} \frac{\mathrm{dT}}{\mathrm{T}}\left(\frac{1}{4 \pi \mathrm{T}}\right)^{\frac{d}{2}} \mathrm{~T}^{\mathrm{N}_{1}+\mathrm{N}_{2}} \exp \left[-m^{2} \mathrm{~T}-\frac{P \circ P}{4 \mathrm{~T}}\right] \\
& \times\left(\prod_{r=1}^{\mathrm{N}_{1}} \int_{0}^{1} d \tau_{r}\right) \exp \left(-\frac{i}{2} \sum_{r<s} p_{r} \wedge p_{s} \varepsilon\left(\tau_{r s}\right)+i p_{r} \wedge p_{s} \tau_{r s}\right) \\
& \times\left(\prod_{a=1}^{\mathrm{N}_{2}} \int_{0}^{1} d \tau_{a}\right) \exp \left(+\frac{i}{2} \sum_{a<b} p_{a} \wedge p_{b} \varepsilon\left(\tau_{a b}\right)-i p_{a} \wedge p_{b} \tau_{a b}\right) \\
& \times \exp \left(\mathrm{T} \sum_{i<j} p_{i a} G^{a b} p_{j b}\left(\left|\tau_{i}-\tau_{j}\right|-\left(\tau_{i}-\tau_{j}\right)^{2}\right)\right)
\end{aligned}
$$

where we have rescaled $\tau$ 's by $\mathrm{T}$ so that they take values in the interval $[0,1]$. The amplitude expression Eq.(3.4) is essentially identical to four point amplitudes $\left(\mathrm{N}_{1}+\mathrm{N}_{2}=4\right)$ obtained in [16] in the case of the $\mathcal{N}=4$ noncommutative $U(1)$ gauge theory up to a number of details. First, the polarization dependence of the gauge fields are deleted in the scalar field theory case. Secondly, $(-1)^{\mathrm{N}_{1}}$ factor is absent reflecting the difference in the parity under $\tau \rightarrow-\tau$ between the tachyon vertex operator (with even worldsheet oscillation number) and the gauge vertex operator (with odd worldsheet oscillation number). Third, while we had to rely on the analytic continuation to make the $m$ value an appropriate number for the scalar theory, one can rely on Higgs mechanism, i.e., the separation $r$ between two parallel D-branes, to produce the mass $m=r /\left(2 \pi \alpha^{\prime}\right)$ for the gauge theories. One further notes that the summation over $\mathrm{N} ! /\left(\mathrm{N}_{1} ! \mathrm{N}_{2} !\right)$ terms fully symmetrizes the external momenta for each nonplanar amplitude, in line with the symmetric trace prescription in nonabelian Born-Infeld theory.

To get further insight into the amplitude Eq.(3.4), we now expand the last line in Eq.(3.4) at low-energy limit:

$$
p_{i a} G^{a b} p_{j b} \ll m^{2} \quad \text { for every } i, j .
$$

In this limit, being subdominant compared to the first line, the last line in Eq.(3.4) simply drops out. The leading term in this expansion exhibits factorization property [17] manifestly:

$$
\begin{aligned}
\Gamma_{\mathrm{N},\left\{\nu_{\mathrm{j}}\right\}=}= & \frac{\hbar}{2}\left(-\frac{\lambda}{2}\right)^{\mathrm{N}} \int_{0}^{\infty} \frac{\mathrm{dT}}{\mathrm{T}}\left(\frac{1}{4 \pi \mathrm{T}}\right)^{\frac{d}{2}} \mathrm{~T}^{\mathrm{N}_{1}+\mathrm{N}_{2}} \exp \left[-m^{2} \mathrm{~T}-\frac{P \circ P}{4 \mathrm{~T}}\right] \\
& \times\left(\prod_{r=1}^{\mathrm{N}_{1}} \int_{0}^{1} d \tau_{r}\right) \exp \left(-\frac{i}{2} \sum_{r<s} p_{r} \wedge p_{s} \varepsilon\left(\tau_{r s}\right)+i p_{r} \wedge p_{s} \tau_{r s}\right) \\
& \times\left(\prod_{a=1}^{\mathrm{N}_{2}} \int_{0}^{1} d \tau_{a}\right) \exp \left(+\frac{i}{2} \sum_{a<b} p_{a} \wedge p_{b} \varepsilon\left(\tau_{a b}\right)-i p_{a} \wedge p_{b} \tau_{a b}\right)
\end{aligned}
$$


The effective action is then obtained by computing the moduli parameter integrals explicitly and then summing over $\Gamma_{\mathrm{N},\left\{\nu_{j}\right\}}$ along with the combinatoric weight $1 / \mathrm{N}$ !, as explained above Eq.(2.7). We elaborate the details in the next section.

\section{Effective Action, $\star_{n}$-products and open Wilson lines}

Begin with evaluation of the moduli parameter integrals of the factorized low-energy expression, Eq.(3.5). As elaborated in the previous section, by rescaling the vertex position moduli $\tau$ 's by $\mathrm{T} \cdot \tau$, the moduli integrals in $\mathrm{T}, \tau_{r}, \tau_{a}$ are also factorized. As such, we evaluate first the T-modulus integral. Recall that the T-modulus corresponds, in the open string worldsheet computation, to the modulus of annulus diagram. One readily obtains

$$
\begin{aligned}
\mathcal{K}_{\mathrm{N}}(P, \Lambda ; d) & :=\int_{0}^{\infty} \frac{\mathrm{dT}}{\mathrm{T}}\left(\frac{1}{4 \pi \mathrm{T}}\right)^{\frac{d}{2}} \mathrm{~T}^{\mathrm{N}} \exp \left[-m^{2} \mathrm{~T}-\frac{P \circ P+\Lambda^{-2}}{4 \mathrm{~T}}\right] \\
& =\left(\frac{1}{2 \pi}\right)^{\frac{d}{2}} 2^{-\mathrm{N}-1}\left(\frac{P \circ P+\Lambda^{-2}}{m^{2}}\right)^{\frac{\mathrm{N}}{2}-\frac{d}{4}} \mathbf{K}_{\mathrm{N}-\frac{d}{2}}\left(m\left|P \circ P+\Lambda^{-2}\right|^{\frac{1}{2}}\right),
\end{aligned}
$$

where we have introduced the UV cutoff $\Lambda$ explicitly, and dependence on $P, \Lambda$, and spacetime dimension $d$ are emphasized. The function $\mathbf{K}_{\frac{d}{2}-\mathrm{N}}(z)$ refers to the modified Bessel function. For the planar diagrams, inferred from Eq.(3.3), $P=0$ and hence the UV cutoff $\Lambda$ is indispensible.

Next, evaluate the moduli integrals in the second and the third lines in Eq.(3.5). These integrals turn out to be identical to the definition of generalized $\star_{\mathrm{N}}$-products, as defined, for instance, in [12]. Note that we have decomposed $\mathrm{N}$-point interaction vertices into $\mathrm{N}_{1}$ untwisted ones and $\mathrm{N}_{2}$ twisted ones, where $\mathrm{N}=\mathrm{N}_{1}+\mathrm{N}_{2}$. In the string worldsheet computation, the former type of insertions corresponds to the 'outer' boundary insertions, and the latter to the 'inner' boundary insertions. One readily finds that each group of the insertions yields a cluster of the generalized $\star$-products. The T-integral, $\mathcal{K}_{\mathrm{N}}(P, \Lambda ; d)$, then supplies a sort of 'propagator', connecting the two clusters of generalized $\star$-products. See figure 1.

As emphasized already, the generalized $\star$-product arises when there exists a net momentum flow between the two clusters of external lines, viz. between untwisted and twisted interacton vertices. As denoted in Eq.(3.3), the net momentum flow $P$ is given by

$$
P+p_{1}+\cdots+p_{\mathrm{N}_{2}}=0
$$

Making use of the identity

$$
\sum_{a<b=1}^{\mathrm{N}_{2}} p_{a} \wedge p_{b}\left(\tau_{a}-\tau_{b}\right)=\sum_{a=1}^{\mathrm{N}_{2}} P \wedge p_{a} \tau_{a},
$$


we can re-express the third line of Eq.(3.5) as

$$
\begin{aligned}
& \left(\prod_{a=1}^{\mathrm{N}_{2}} \int_{0}^{1} d \tau_{a}\right) \exp \sum_{a<b=1}^{\mathrm{N}_{2}}\left(\frac{i}{2} \varepsilon\left(\tau_{a b}\right) p_{a} \wedge p_{b}-i \tau_{a b} p_{a} \wedge p_{b}\right) \\
= & \left(\prod_{a=1}^{\mathrm{N}_{2}} \int_{0}^{1} d \tau_{a}\right) \exp \sum_{a<b=1}^{\mathrm{N}_{2}}\left(\frac{i}{2} \varepsilon\left(\tau_{a b}\right) p_{a} \wedge p_{b}\right) \exp \left(-i \sum_{a} P \wedge p_{a} \tau_{a}\right) .
\end{aligned}
$$

As expressed, the moduli integrals over $\tau$ 's are unordered and ranges over the entire circle $[0,1]$. One can decompose these integrals into $\mathrm{N}_{2}$ ! ordered integrals, each of which is defined with a definite ordering among the $\mathrm{N}_{2} \tau_{a}$-moduli parameters. For each ordering, the first exponential in Eq.(4.2) gives rise to Filk's phase-factor, which, in the absence of the second exponential, simply yields the standard $\star$-product. In the case of the planar contribution, $P=0$ and the relevant product is the symmetrized form of the standard $\star$-product:

$$
\left[A_{1} A_{2} \cdots A_{\mathrm{N}}\right]_{\star_{\mathrm{sym}}}:=\frac{1}{\mathrm{~N} !} \sum_{\{\mathrm{perm}\}} A_{i 1} \star \cdots \star A_{i \mathrm{~N}}
$$

where the summation is over N!-permutations. In the case of the nonplanar contributions, however, because of nonvanishing momentum flow $P$, the relevant product turns out to be the generalized $\star_{\mathrm{N}}$-product [

The explicit evaluation of Eq.(4.2), including the nonabelian Chan-Paton factor, was made in [17]. The results are:

$$
\begin{aligned}
& \operatorname{Tr}\left[f_{1}\left(p_{1}\right), f_{2}\left(p_{2}\right), \cdots f_{\mathrm{N}_{2}}\left(p_{\mathrm{N}_{2}}\right)\right]_{\star_{\mathrm{N}_{2}}}=\sum_{\left(\mathrm{N}_{2}-1\right) !} f_{1}^{a_{1}}\left(p_{1}\right) \cdots f_{\mathrm{N}_{2}}^{a_{\mathrm{N}_{2}}}\left(p_{\mathrm{N}_{2}}\right) \operatorname{Tr}\left(\mathrm{T}^{a_{1}} \cdots \mathrm{T}^{a_{\mathrm{N}_{2}}}\right) \\
& \times\left(\frac{\exp \left[\frac{i}{2} \sum_{i<j}^{\mathrm{N}_{2}} p_{i} \wedge p_{j}\right]}{\prod_{i=2}^{\mathrm{N}_{2}}\left(-i k \wedge P_{i}\right)}+(\text { cyclic permutations })\right),
\end{aligned}
$$

where the summation runs over the $\left(\mathrm{N}_{2}-1\right)$ ! noncyclic permutations (with independent ChanPaton factor), $f_{i}=\sum_{a_{i}} f_{i}^{a_{i}} \mathrm{~T}^{a_{i}}, \mathrm{~T}^{a_{i}}$ are generators of the $U(n)$ Chan-Paton group, and $P_{i}:=$ $\sum_{j=i}^{\mathrm{N}} p_{j}$. For $U(1)$ gauge group, they reduce to:

$$
\begin{aligned}
{\left[f_{1}\left(p_{1}\right), f_{2}\left(p_{2}\right), \cdots f_{\mathrm{N}_{2}}\left(p_{\mathrm{N}_{2}}\right)\right]_{\mathrm{N}_{2}} } & =\sum_{\left(\mathrm{N}_{2}-1\right) !} f_{1}\left(p_{1}\right) \cdots f_{\mathrm{N}_{2}}\left(p_{\mathrm{N}_{2}}\right) \\
& \times\left(\frac{\exp \left[\frac{i}{2} \sum_{i<j}^{\mathrm{N}_{2}} p_{i} \wedge p_{j}\right]}{\prod_{i=2}^{\mathrm{N}_{2}}\left(-i k \wedge P_{i}\right)}+(\text { cyclic permutations })\right) .
\end{aligned}
$$

\footnotetext{
${ }^{3}$ Relevance of generalized $\star$-products and relation to gauge invariance and Seiberg-Witten map have been studied recently [18], but all in the context of noncommutative gauge theories.
} 
One can explicitly work out and find that they are given by

$$
\begin{gathered}
{\left[A\left(x_{1}\right) B\left(x_{2}\right)\right]_{\star_{2}}:=\frac{\sin \left(\frac{1}{2} \partial_{1} \wedge \partial_{2}\right)}{\frac{1}{2} \partial_{1} \wedge \partial_{2}} A\left(x_{1}\right) B\left(x_{2}\right)} \\
{\left[A\left(x_{1}\right) B\left(x_{2}\right) C\left(x_{3}\right)\right]_{\star_{3}}:=\left[\frac{\sin \left(\frac{1}{2} \partial_{2} \wedge \partial_{3}\right)}{\frac{1}{2}\left(\partial_{1}+\partial_{2}\right) \wedge \partial_{3}} \frac{\sin \left(\frac{1}{2} \partial_{1} \wedge\left(\partial_{2}+\partial_{3}\right)\right)}{\frac{1}{2} \partial_{1} \wedge\left(\partial_{2}+\partial_{3}\right)}+(1 \leftrightarrow 2)\right] A\left(x_{1}\right) B\left(x_{2}\right) C\left(x_{3}\right)}
\end{gathered}
$$

and so forth. Evidently, as $k$ subset of momenta go to zero, $\star_{\mathrm{N}}$ is reduced to $\star_{\mathrm{N}-k}$.

Combining Eq.(4.1) and Eq.(4.3), Eq.(3.5) can be rewritten as

$$
\Gamma_{\mathrm{N},\left\{\nu_{\mathrm{j}}\right\}}=\frac{\hbar}{2}\left(-\frac{\lambda}{4}\right)^{\mathrm{N}_{1}+\mathrm{N}_{2}}[\Phi \cdots \Phi]_{\star_{\mathrm{N}_{1}}}(-P) \widetilde{\mathcal{K}}_{\mathrm{N}-d / 2}\left(P \circ P+1 / \Lambda^{2}\right)[\Phi \cdots \Phi]_{\star_{\mathrm{N}_{2}}}(+P)
$$

where the kernel $\mathcal{K}_{n}$ is given, from Eq.(4.1), by

$$
\tilde{\mathcal{K}}_{n}\left(z^{2}\right)=2\left(\frac{1}{2 \pi}\right)^{\frac{d}{2}}\left(\frac{|z|}{m}\right)^{n} \mathbf{K}_{n}(m|z|) .
$$

In Eq.(4.4), we have retained the UV cutoff $\Lambda$, as the result is equally valid for planar contributions in so far as $P$ is set to zero and the generalized $\star$-products are replaced by the standard *-products.

As is clear from the defintion in Eq.(4.3), the generalized star products are symmetric with respect to the external momenta. Hence, each summand in the $2^{\mathrm{N}_{1}+\mathrm{N}_{2}}$-summations over $\left\{\nu_{j}\right\}$ yield the same contribution as long as $\mathrm{N}_{1}$ (thus $\mathrm{N}_{2}$ ) is the same; this gives rise to the combinatoric factor $\mathrm{N} ! /\left(\mathrm{N}_{1} ! \mathrm{N}_{2}\right.$ !). Recalling the definition of the effective action in our convention with the $1 / \mathrm{N}$ ! factor, we finally obtain the one-loop effective action as

$$
\begin{aligned}
\Gamma[\Phi]= & \frac{\hbar}{2} \sum_{\mathrm{N}=1}^{\infty}\left(-\frac{\lambda}{4}\right)^{\mathrm{N}} \frac{1}{\mathrm{~N} !} \int \mathrm{d}^{d} x \sum_{\mathrm{N}_{1}=0}^{\mathrm{N}} \frac{\mathrm{N} !}{\mathrm{N}_{1} !\left(\mathrm{N}-\mathrm{N}_{1}\right) !} \\
& \times[\Phi \cdots \Phi]_{\star_{\mathrm{N}}}(x) \widetilde{\mathcal{K}}_{\mathrm{N}-d / 2}\left(-\partial_{x} \circ \partial_{x}+\Lambda^{-2}\right)[\Phi \cdots \Phi]_{\star_{\mathrm{N}-\mathrm{N}_{1}}}(x),
\end{aligned}
$$

encompassing both planar $\left(\mathrm{N}_{1}=0\right.$ or $\left.\mathrm{N}\right)$ and nonplanar contributions. As we will see below, the planar contribution is the counterpart of the closed Wilson loop one-point function, viz. counterpart of the closed string tadpole, while the nonplanar contribution is the counterpart of the open Wilson line two-point functions. See figure 4.

\subsection{Planar Contribution}

We first consider the planar contribution $\mathrm{N}_{1}=0$ or $\mathrm{N}$ in the limit where the momentum cutoff is much larger than the mass scale $m, \Lambda \gg m$. From the Taylor expansion of the modified Bessel function, we obtain the following expressions for the kernel:

$$
\mathcal{K}_{n}\left(z^{2}\right)=2\left(\frac{1}{2 \pi}\right)^{d / 2}\left(\frac{|z|}{m}\right)^{n} \frac{\Gamma(|n|)}{2}\left(\frac{2}{m|z|}\right)^{|n|}
$$




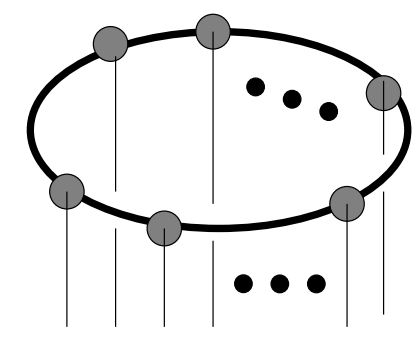

$\mathrm{P}=0$

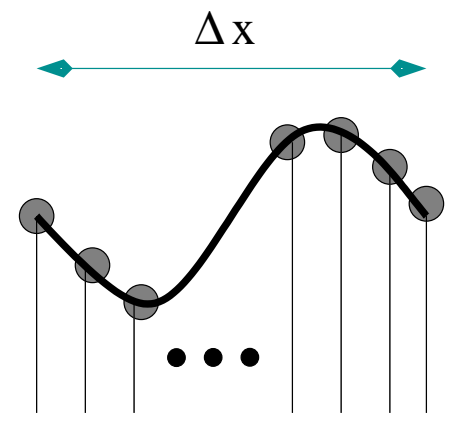

$\mathrm{P} \neq 0$

Figure 4: Spacetime snapshot of planar and nonplanar contributions. Partial momentum sum of (un)twisted interaction vertices is denoted as $P$. For $P=0$, viz. planar contribution, the virtual quanta sweeps a closed path in spacetime. When $P \neq 0$, viz. nonplanar contribution, the virtual quanta jumps $\Delta x$ in spacetime.

for $n \neq 0$ and

$$
\mathcal{K}_{0}\left(z^{2}\right)=2\left(\frac{1}{2 \pi}\right)^{\frac{d}{2}}\left(-\log \frac{m|z|}{2}\right)
$$

for $n=0$ and $n=\mathrm{N}-d / 2$. Here, $z=1 / \Lambda$, and, as explained above, the generalized star products should be understood as the standard star products:

$$
[\Phi, \cdots, \Phi]_{\star_{\mathrm{N}_{1}}}(x)[\Phi, \cdots, \Phi]_{\star_{\mathrm{N}-\mathrm{N}_{1}}}(x)=[\Phi \star \cdots \star \Phi](x)
$$

viz. N-tuple of the standard $\star$-products, etc. Details of the UV divergence depends on the spacetime dimension $d$. For instance, in $d=6$ wherein the theory is renormalizable, the twopoint Green function $(\mathrm{N}=2)$ is quadratically divergent, and the three-point Green function $(\mathrm{N}=3)$ is logarithmically divergent. The higher-point functions $(n>0)$ are finite, as dependence on the UV cutoff $\Lambda$ cancels out. One furthermore observes that, after renormalization of the divergent contributions, the combinatorics factors come out as

$$
\frac{(\mathrm{N}-4) !}{\mathrm{N} !}=\frac{1}{\mathrm{~N}(\mathrm{~N}-1)(\mathrm{N}-2)(\mathrm{N}-3)}=-\frac{1}{6}\left(\frac{1}{\mathrm{~N}}-\frac{3}{\mathrm{~N}-1}+\frac{3}{\mathrm{~N}-2}-\frac{1}{\mathrm{~N}-3}\right) .
$$

As such, the planar contribution to the effective action is given by:

$$
\Gamma_{\mathrm{p}} \simeq \hbar\left(m^{2}+\frac{\lambda}{2} \Phi\right)_{\star}^{3} \star \log _{\star}\left(m^{2}+\frac{\lambda}{2} \Phi\right) .
$$

The result Eq.(4.5) is precisely noncommutative version of the 'Coleman-Weinberg'-type potential, where the parameters and the fields are to be understood as renormalized ones.

The planar contribution ought to correspond, in string theory, to the diagrams with a tadpole insertion [19]. This is evidently so, except one puzzling point: in the large noncommutativity limit, the Weyl-Moyal correspondence permits to map the noncommutative $\lambda\left[\Phi^{3}\right]_{\star}$-theory 
into large- $N$ limit of $\mathrm{U}(N)$ matrix $\lambda \operatorname{Tr}[\Phi]^{3}$-theory. One would have expected that the dominant dynamics is describable in terms of the standard Wilson loop operators

$$
W_{0}[\Phi]:=\int \mathrm{d}^{4} x \exp _{\star}(-\lambda \Phi(x)),
$$

where the integration is over the noncommutative directions. Typically, these Wilson loops are the large- $N$ limit 'master' fields in matrix-valued field theories. Apparently, the result, Eq.(4.5), does not involve the above Wilson loops, even after taking the large noncommutativity limit. Whether this discrepancy invalidates the concept of master field in this context or not is unclear yet.

\subsection{Nonplanar Contribution}

The behavior of the nonplanar contribution is markedly different from those of the planar part, especially as we take the large noncommutativity limit. From here on, we will drop the cutoff by sending it to infinity and Wick-rotate back to the Minkowski space. The nonplanar part of the effective action then becomes a double sum involving the generalized $\star$-products:

$$
\Gamma_{\mathrm{np}}=\frac{\hbar}{2} \sum_{\mathrm{N}=2}^{\infty}\left(-\frac{\lambda}{4}\right)^{\mathrm{N}} \frac{1}{\mathrm{~N} !} \int \mathrm{d}^{d} x \sum_{n=1}^{\mathrm{N}-1}\left(\begin{array}{l}
\mathrm{N} \\
n
\end{array}\right)[\Phi \cdots \Phi]_{\star_{\mathrm{n}}}(x) \mathcal{K}_{\mathrm{N}-\frac{d}{2}}\left(-\partial_{x} \circ \partial_{x}\right)[\Phi \cdots \Phi]_{\star_{\mathrm{N}-n}}(x) .
$$

To proceed further, we will be taking the low-energy, large noncommutativity limit:

$$
q_{\ell} \sim \epsilon, \quad \operatorname{Pf} \theta \sim \frac{1}{\epsilon^{2}} \quad \text { as } \quad \epsilon \rightarrow 0
$$

so that

$$
q_{\ell} \cdot q_{m} \sim \mathcal{O}\left(\epsilon^{+2}\right) \rightarrow 0, \quad q_{\ell} \wedge q_{m} \rightarrow \mathcal{O}(1), \quad q_{\ell} \circ q_{m} \sim \mathcal{O}\left(\epsilon^{-2}\right) \rightarrow \infty
$$

In this limit, the modified Bessel function $\mathbf{K}_{n}$ exhibits the following asymptotic behavior:

$$
\mathbf{K}_{n}(m z) \rightarrow \sqrt{\frac{\pi}{2 m z}} e^{-m|z|}\left[1+\mathcal{O}\left(\frac{1}{m|z|}\right)\right]
$$

Most remarkably, the asymptotic behavior is independent of the index $n$. Compared to the planar effective action, there is not the extra $n$ ! factor that (partially) cancels $\mathrm{N}$ ! in the denominator, which shows that the summed form of the effective action markedly changes. In the low-energy limit, the Fourier-transformed kernels, $\widetilde{\mathcal{K}_{n}}$, obey the following recursive relation:

$$
\widetilde{\mathcal{K}}_{n+1}(k \circ k)=\left(\frac{|\theta \cdot k|}{m}\right) \widetilde{\mathcal{K}}_{n}(k \circ k),
$$

viz.

$$
\widetilde{\mathcal{K}_{n}}(k \circ k)=\left(\frac{|\theta \cdot k|}{m}\right)^{n} \widetilde{\mathcal{Q}}(k \circ k)
$$


Here, the kernel $\widetilde{\mathcal{Q}}$ is given by:

$$
\widetilde{\mathcal{Q}}(k \circ k)=(2 \pi)^{(1-d) / 2}\left|\frac{1}{m \theta \cdot k}\right|^{\frac{1}{2}} \exp (-m|\theta \cdot k|) .
$$

Note that, in the power-series expansion of the effective action, a natural expansion parameter is $|\theta \cdot k|$.

Thus, the nonplanar one-loop effective action in momentum space is expressible as:

$$
\begin{aligned}
\Gamma_{\mathrm{np}}[\Phi] & =\frac{\hbar}{2} \int \frac{\mathrm{d}^{d} k}{(2 \pi)^{d}} \widetilde{\mathcal{K}}_{-\frac{d}{2}}(k \circ k) \sum_{\mathrm{N}=2}^{\infty} \sum_{n=1}^{\mathrm{N}-1}\left(-\frac{\lambda}{4 m}\right)^{\mathrm{N}} \\
& \times\left(\frac{1}{n !}|\theta \cdot k|^{n}[\widetilde{\Phi} \ldots \widetilde{\Phi}]_{\star_{n}}[k]\right)\left(\frac{1}{(\mathrm{~N}-n) !}|\theta \cdot k|^{\mathrm{N}-n}[\widetilde{\Phi} \ldots \widetilde{\Phi}]_{\star_{\mathrm{N}-n}}[-k]\right) .
\end{aligned}
$$

Utilizing the relation between the generalized $\star_{n}$ products and the scalar open Wilson line operators, as elaborated in [1], the nonplanar one-loop effective action can be summed up into a remarkably simple closed form. Denote the rescaled coupling parameter as $g:=\lambda / 4 m$ (see Eq.(1.2)). Then, because of the algebraic relation $\left[\widetilde{\Phi} \star_{0} \widetilde{\Phi}\right]_{k}=(2 \pi)^{d} \delta^{(d)}(k)$, domain of the double summations can be extended to $n=0, \mathrm{~N}=0$ terms, as they yield identically vanishing contribution after $k$-integration is performed. Once this arrangement is made, partial summations over $\mathrm{N}$ and $n$ can be performed explicitly. Exploiting the exchange symmetry $n \leftrightarrow$ $(\mathrm{N}-n)$, the summation domain $(n, \mathrm{~N})$ over the lower triangular lattice points can be mapped to the one over the upper triangular lattice points. By averaging over the two summation domains, one can then rearrange the double summations into decoupled ones over $n$ and $(\mathrm{N}-n)$. One finally obtains:

$$
\Gamma_{\mathrm{np}}[\Phi]=\frac{\hbar}{2} \int \frac{\mathrm{d}^{d} k}{(2 \pi)^{d}} W_{k}[\Phi] \cdot \widetilde{\mathcal{K}_{-\frac{d}{2}}}(k \circ k) \cdot W_{-k}[\Phi],
$$

yielding precisely the aforementioned result, Eq.(1.1).

\section{Conclusions and Discussions}

In this paper, we have studied the one-loop effective action in the noncommutative $\lambda\left[\Phi^{3}\right]_{\star^{-}}$ theory. In order to make direct comparison with the Seiberg-Witten limit of the open string worldsheet formulation, in computing the one-particle irreducible one-loop Green functions, we have adopted the worldline formulation of the theory. We have observed that, at lowenergy, the one-loop diagrams, both planar and nonplanar, are completely factorizable. We have shown explicitly that, while the planar contribution is expressed in terms of the standard $\star-$ products, the nonplanar contribution is expressible solely in terms of the generalized $\star$-products. This implies that structure of the one-loop effective action reveals quite a different physics 
between planar and nonplanar contributions. In particular, we were able to show that, at large noncommutativity limit, the nonplanar contribution is expressible in terms of open Wilson line operators, thus completing the proof of our earlier result in [1]. The planar contribution, on the other hand, gives rise to a noncommutative version of the Coleman-Weinberg type potential, in contrast to the anticipation that the planar part ought to be expressible in terms of Wilson loop opeators - the putative master field in planar limit of matrix-valued field theories. The next obvious step is to extend the computation to two-loop and confirm that two-loop effective action is re-expressible in terms of (at most) three open Wilson line operators. We will report the result in a separate publication.

Our computation in the worldline formulation has revealed several new light concerning spacetime interpretation of the one-loop physics. Among them is concerning the shift of the momentum integration variable, as is evident from integration $K$ in section 2.2. It suggests that variation of the internal momemtum $\Delta k^{a}$ integrated along the entire loop amounts to

$$
\begin{aligned}
\Delta \int_{0}^{\mathrm{T}} \mathrm{d} \tau k^{a}(\tau) & =\frac{i}{2} \int_{0}^{\mathrm{T}} \mathrm{d} \tau\left\{\dot{x}^{a}(\tau)-\frac{1}{2} \sum_{j=1}^{\mathrm{N}} \theta^{a b} p_{b j} \nu_{j} \delta\left(\tau-\tau_{j}\right)\right\} \\
& =\frac{i}{2} \sum_{j=1}^{\mathrm{N}} \theta^{a b} p_{b j} \varepsilon_{j} .
\end{aligned}
$$

Recalling $\varepsilon_{j}$ take either 0 or 1 , depending on whether the $j$-th vertex is untwisted or twisted insertion, we recognize that the above relation is precisely the momentum-space counterpart of the dipole relation Eq.(1.3). Recalling that $k^{a}(\tau)$ is the conjugate momentum to $x^{a}(\tau)$, both of which are associated with the virtual quanta circulating around the loop, the above relation asserts that the virtual quanta is not a point-like constituent, obeying the standard Fourier transformation relation between $x^{a}(\tau)$ and $k^{a}(\tau)$, but behaves as a sort of rigid rod whose size is proportioanl to the momentum. We trust details of this unusual physics - physics of dipoles - deserve further investigation and intend to report new understanding concerning this aspect in separate publications.

\section{Acknowledgement}

We thank H. Liu, P. Mayr, Y. Oz, and L. Susskind for discussions. SJR acknowledges warm hospitality of Henri Poincaré Institut, Institut des Hautes Etudes Scientifiques, Theory Division at CERN, and Department of Mathematics and Physics at Amsterdam University at various stage of this project.

\section{Appendix}




\section{A Schwinger parametrization of one-loop Feynman dia- gram}

In this section, we will provide a check point of the worldline formulation introduced in sections 2 with the standard Feynman diagrammatics. Although a general expression for Npoint, one-loop Feynman diagrams are given, for instance, in [20] or in [9], they are not in a convenient form for comparison with the results in the worldline formulation, mainly because of different moduli parametrizations and omission of overall normalization and combinatoric factors (which are necessary for resummation of the N-point Green functions into the effective action). Let us consider the N-point Feynman diagram (cf. Fig. 2), wherein the nonplanar phase-factors $e^{i k \wedge p_{1}}, e^{i\left(k+p_{1}\right) \wedge p_{2}}, e^{i\left(k+p_{1}+p_{2}\right) \wedge p_{3}}, \cdots, e^{i k \wedge p_{\mathrm{N}}}$ are inserted at each of the $\mathrm{N}$ interaction vertices, respectively, as well as the overall Filk's phase-factor $e^{-\frac{i}{2} \sum_{i<j} p_{i} \wedge p_{j}}$. The one-loop Feynman amplitude is given by

$$
F_{\mathrm{N}}=e^{-\frac{i}{2} \sum_{i<j} p_{i} \wedge p_{j}} \int \frac{\mathrm{d}^{d} k}{(2 \pi)^{d}} \frac{e^{i k \wedge P} e^{i \sum_{l=2}^{\mathrm{N}-1} \sum_{i=2}^{l-1} p_{i} \wedge p_{l} \varepsilon_{l}}}{k^{2}\left(k+p_{1}\right)^{2}\left(k+p_{1}+p_{2}\right)^{2} \cdots\left(k+p_{1}+p_{2}+\cdots+p_{\mathrm{N}-1}\right)^{2}} .
$$

Here, the twist factor $\varepsilon_{i}=1$ or $0 ; i=1, \cdots, \mathrm{N}$ are inserted for the planar the and nonplanar vertex insertions, respectively, and

$$
P^{a}=\sum_{i=1}^{\mathrm{N}} \varepsilon_{i} p_{i}^{a}
$$

We rewrite the momentum integral in terms of an overall modulus integral (global Schwinger parameter $\left.T \equiv \tau_{\mathrm{N}}\right)$ and $(\mathrm{N}-1)$ relative moduli integrals (local Schwinger parameters, $\left.\tau_{i}\right)$ :

$$
\begin{aligned}
F_{\mathrm{N}} & =\prod_{i<j=1}^{\mathrm{N}} e^{-\frac{i}{2} p_{i} \wedge p_{j}} \prod_{k=2}^{\mathrm{N}-1} \prod_{\ell=2}^{k-1} e^{i p_{\ell} \wedge p_{k} \varepsilon_{k}} \\
& \times \int_{0}^{\infty} \mathrm{dT}\left(\frac{1}{4 \pi \mathrm{T}}\right)^{\frac{d}{2}} \prod_{n=1}^{\mathrm{N}-1} \int_{0}^{\tau_{n+1}} \mathrm{~d} \tau_{n} \exp \left[\frac{1}{\mathrm{~T}}\left(\sum_{j=1}^{\mathrm{N}-1} \tau_{j j+1} \sum_{\ell=1}^{j} p_{\ell}+\frac{i}{2} \theta \cdot P\right)^{2}-\sum_{j=1}^{\mathrm{N}-1} \tau_{j j+1}\left(\sum_{\ell=1}^{j} p_{\ell}\right)^{2}\right]
\end{aligned}
$$

where $\tau_{i j}=\tau_{i}-\tau_{j}$. From the energy-momentum conservation, the following relations can be deduced:

$$
\begin{gathered}
P \circ P=-\sum_{i<j}^{\mathrm{N}} \varepsilon_{i j}^{2} p_{i} \circ p_{j}, \\
\sum_{j=1}^{\mathrm{N}-1} \tau_{j j+1} \sum_{l=1}^{j} p_{\ell} \wedge P=\sum_{i<j}^{\mathrm{N}}\left(\tau_{i}+\tau_{j}\right) \varepsilon_{j i} p_{i} \wedge p_{j}=-\sum_{i<j}^{\mathrm{N}} \sum_{k=1, k \neq i, j}^{\mathrm{N}} \tau_{k} \varepsilon_{j i} p_{i} \wedge p_{j}, \\
\frac{1}{\mathrm{~T}}\left(\sum_{j=1}^{\mathrm{N}-1} \tau_{j j+1} \sum_{\ell=1}^{j} p_{\ell}\right)^{2}-\sum_{j=1}^{\mathrm{N}-1} \tau_{j j+1}\left(\sum_{\ell=1}^{j} p_{\ell}\right)^{2}=\sum_{i<j}^{\mathrm{N}} p_{i} \cdot \mathcal{G}_{B}\left(\tau_{j}, \tau_{i}\right) \cdot p_{j},
\end{gathered}
$$


and

$$
\sum_{i<j}^{\mathrm{N}} \varepsilon_{i j} p_{i} \wedge p_{j}=\sum_{i<j}^{\mathrm{N}}\left(\nu_{i}-\nu_{j}\right) p_{i} \wedge p_{j}=\sum_{i<j}^{\mathrm{N}} \tau_{i j} p_{i} \wedge p_{j}=0
$$

Utilizing the identity Eq.(A.4), the nonplanar phase-factor can be simplified as

$$
e^{i \sum_{l=2}^{\mathrm{N}-1} \sum_{i=2}^{l-1} p_{i} \wedge p_{l} \varepsilon_{l}}=e^{i \sum_{i<j} \varepsilon_{i} p_{i} \wedge p_{j}},
$$

thereby deriving

$$
F_{\mathrm{N}}=e^{-\frac{i}{2} \sum_{i<j} \nu_{j} p_{i} \wedge p_{j}} \int_{0}^{\infty} \mathrm{dT}\left(\frac{1}{4 \pi \mathrm{T}}\right)^{\frac{d}{2}} \prod_{n=1}^{\mathrm{N}-1} \int_{0}^{\tau_{n+1}} \mathrm{~d} \tau_{n} \exp \left[\sum_{i<j}^{\mathrm{N}} p_{i} \cdot \mathcal{G}_{B \theta}\left(\tau_{i}, \tau_{j} ; \varepsilon_{i}, \varepsilon_{j}\right) \cdot p_{j}\right] .
$$

Using the above identities again, Filk's overall phase-factor in Eq.(A.5) can be reduced to

$$
-\frac{i}{2} \sum_{i<j} p_{i} \wedge p_{j} \nu_{j}=-\frac{i}{2} \sum_{i<j} p_{i} \wedge p_{j} \frac{1}{2}\left(\nu_{i}+\nu_{j}\right)
$$

and

$$
\begin{aligned}
F_{\mathrm{N}}= & \int_{0}^{\infty} \mathrm{dT}\left(\frac{1}{4 \pi \mathrm{T}}\right)^{\frac{d}{2}} e^{-m^{2} \mathrm{~T}} \prod_{n=1}^{\mathrm{N}-1} \int_{0}^{\tau_{n+1}} \mathrm{~d} \tau_{n} \prod_{i<j}^{\mathrm{N}} \exp \left(\frac{i}{2} p_{i} \wedge p_{j} \frac{1}{2}\left(\nu_{i}+\nu_{j}\right) \varepsilon\left(\tau_{j i}\right)\right) \\
& \times \exp \left[\sum_{i<j}^{\mathrm{N}} p_{i} \cdot \mathcal{G}_{B \theta}\left(\tau_{i}, \tau_{j} ; \varepsilon_{i}, \varepsilon_{j}\right) \cdot p_{j}\right] .
\end{aligned}
$$

Here, having in mind of generalization to all possible orderings, we have attached $\varepsilon\left(\tau_{j i}\right)$ to the phase-factor in order to blindly count the ordering dependences. If we shuffle the external legs, we obtain different diagrams with different phase-factors. Thus all ordered integrals correspond to all possible distinct Feynman diagrams. In this way, we recover the full integral regions after summing all contributions for fixed $\nu_{i}$. Obviously, we then also have to sum over all combinations of $\nu_{i}$. As, in section 2.2, Eq.(2.8) is invariant under the simultaneous constant shifts of all $\tau$ (by noticing Eq.(2.9) and Eq.(A.4)), we can fix one of them in Eq.(2.8); for example, $\tau_{\mathrm{N}}=\mathrm{T}$, as has been assumed throughout this section, and reproduce

$$
\begin{aligned}
\Gamma_{\mathrm{N}}= & C(-\lambda)^{\mathrm{N}} \sum_{\nu_{j}} \int \mathrm{d} \mathrm{T} e^{-m^{2} \mathrm{~T}}\left(\frac{1}{4 \pi \mathrm{T}}\right)^{\frac{d}{2}}\left(\prod_{i=1}^{\mathrm{N}-1} \int_{0}^{\mathrm{T}} \mathrm{d} \tau_{i}\right) e^{\frac{i}{4} \sum_{i<j} p_{i} \wedge p_{j}\left(\nu_{i}+\nu_{j}\right) \varepsilon\left(\tau_{i j}\right)} \\
& \times \exp \left[\frac{1}{2} \sum_{i, j=1}^{\mathrm{N}} p_{i} \cdot \mathcal{G}_{B \theta}\left(\tau_{i}, \tau_{j} ; \varepsilon_{i}, \varepsilon_{j}\right) \cdot p_{j}\right]
\end{aligned}
$$

Here, $C$ is the normallization factor defined by a fraction between the symmetry factor $S_{\mathrm{N}}$ and the number of topologically distinct integration regions $C_{\mathrm{N}}$ :

$$
C=\frac{S_{\mathrm{N}}}{C_{\mathrm{N}}}
$$


Following closely the arguments of Ref [21], we will now determine the combinatoric factor $C_{\mathrm{N}}$. If one expands the effective action in powers of the coupling constant, the combinatoric weight for the expansion of the Feynman diagrams is obtained by shuffling the external interaction vertices. One obtains

$$
w=\frac{S_{\mathrm{N}} n^{\mathrm{T}}}{\mathrm{N} !}
$$

where $n^{\mathrm{T}}$ is the number of the topologically distinct diagrams out of those shuffled diagrams, $S$ is the symmetry factor for those. In the present case, this number $w$ is given by

$$
w=\frac{1}{2 \cdot \mathrm{N} \cdot 2^{\mathrm{N}}} .
$$

It comprises of trace-log factor $\frac{1}{2}$, the $\frac{1}{\mathrm{~N}}$ coming from the Taylor expansion of an one-loop form $\ln (1+x)$, and the coupling factor $2^{-\mathrm{N}}$. (One may simply multiply $2^{-\mathrm{N}}$ to the result Eq. $(3.25)$ of Ref [21]). Combining Eq.(A.8) and Eq.(A.9), we obtain

$$
S_{\mathrm{N}}=\frac{(\mathrm{N}-1) !}{2^{\mathrm{N}+1} \mathrm{n}^{\mathrm{T}}}
$$

As $C_{\mathrm{N}}$ is defined by

$$
C_{\mathrm{N}}=\frac{(\mathrm{N}-1) !}{n^{\mathrm{T}}}
$$

we can put these into Eq. A.7) and obtain

$$
C=\frac{1}{2 \cdot 2^{\mathrm{N}}}
$$

therefore proving that our world-line master formula coincides with the Feynman diagrammatics result.

The commutative limit, $\theta^{a b} \rightarrow 0$, also comes out correct, as all the $2^{\mathrm{N}}$ terms of the $\left\{\nu_{i}\right\}$ summation are reduced to the same, single contribution, reproducing the commutative result.

\section{References}

[1] Y. Kiem, S.-J. Rey, H.-T. Sato, and J.-T. Yee, Open Wilson Lines and Generalized Star Product in Noncommutative Scalar Field Theories, to appear in Phys. Rev. D (2001) hep-th/0106121.

[2] S.-J. Rey and R. von Unge, Phys. Lett.B499, 215 (2001) hep-th/0007089.

[3] S.R. Das and S.-J. Rey, Nucl. Phys. B590,453 (2000) hep-th/0008042.

[4] D.J. Gross, A. Hashimoto, and N. Itzhaki, hep-th/0008075. 
[5] N. Ishibashi, S. Iso, H. Kawai and Y. Kitazawa, Nucl. Phys. B573, 573 (2000) hep-th/9910004.

[6] N. Seiberg and E. Witten, J. High-Energy. Phys. 9909, 032 (1999) hep-th/9908142.

[7] A.M. Polyakov, "Gauge Fields and Strings" (Harwood, 1987);

M.J. Strassler, Nucl. Phys. B385, 145 (1992);

M.G. Schmidt and C. Schubert, Phys. Lett. B331, 69 (1994); ibid, Phys. Lett. B318, 438 (1993);

H.T. Sato, Phys. Lett. B371, 270 (1996) 270; ibid, J. Math. Phys. 40, 6407 (1999);

C. Schubert, hep-th/0101036.

[8] T. Filk, Phys. Lett. B376, 53 (1996).

[9] S. Minwalla, M. Van Raamsdonk and N. Seiberg, J. High-Energy Phys. 0002, 020 (2000) hep-th/9912072.

[10] M. Hayakawa, Phys. Lett. B 478, 394 (2000) hep-th/9912094;

A. Matusis, L. Susskind and N. Toumbas, J. High-Energy Phys. 0012, 002 (2000) hep-th/0002075;

M. Van Raamsdonk and N. Seiberg, J. High-Energy Phys. 0003, 035 (2000) hep-th/0002186.

[11] M. R. Garousi, Nucl. Phys. B 579, 209 (2000) hep-th/9909214.

[12] H. Liu, hep-th/0011125.

[13] O. Andreev and H. Dorn, Nucl.Phys. B583, 145 (2000), hep-th/0003113 ;

Y. Kiem and S. Lee, Nucl. Phys. B586, 303 (2000), hep-th/0003145;

A. Bilal, C.-S. Chu and R. Russo, Nucl. Phys. B582, 65 (2000), hep-th/0003180;

H. Liu and J. Michelson, Phys. Rev. D62, 066003 (2000), hep-th/0004013.

[14] Y. Kiem, S. Lee and J. Park, Nucl. Phys. B 594, 169 (2001) hep-th/0008002.

[15] K. Roland and H.T. Sato, Nucl. Phy. B480, 99 (1996); ibid, Nucl. Phys. B515, 488 (1998).

[16] H. Liu and J. Michelson, hep-th/0008205.

[17] Y. Kiem, S. Lee and D.H. Park, Phys. Rev. D63, 126006 (2001), hep-th/0011233.

[18] S. R. Das and S. P. Trivedi, J. High-Energy Phys. 0102, 046 (2001) hep-th/0011131;

Y. Okawa and H. Ooguri, Nucl. Phys. B599, 55 (2001) hep-th/0012218;

H. Liu and J. Michelson, hep-th/0101016; 
S. Mukhi and N. V. Suryanarayana, J. High-Energy Phys. 0105, 023 (2001) 023 hep-th/0104045;

Y. Okawa and H. Ooguri, hep-th/0104036;

S. Mukhi and N. V. Suryanarayana, J. High-Energy Phys. 0105, 023 (2001) hep-th/0104045;

H. Liu and J. Michelson, hep-th/0104139.

[19] S.-J. Rey, Phys. Lett. B 203, 393 (1988) ;

R. Ordonez, S.-J. Rey, M. A. Rubin and R. Zucchini, Phys. Rev. D 40, 1987 (1989).

[20] I. Chepelev and R. Roiban, J. High-Energy Phys. 0005, 037, (2000).

[21] M.G. Schmidt and H.T. Sato, Nucl. Phys. B524, 742 (1998). 